\title{
FACTORIAL ANALYSIS STUDY OF PHYSICAL FLEXIBILITY CONCERNING THE VALIDITY OF THE METHOD USED AND THE SELECTION OF THE MEASURING ITEMS
}

\author{
YOSHINORI OHYAMA*
}

\begin{abstract}
The first object of this study was to investigate the constituent factor of physical flexibility. The second purpose was to reconsider if the measures of physical flexibility which have been customarily used are valid, and choose those measures which have high validity. To investigate this the method of factor analysis was used.

The following results were obtained:

1) It has been recognized that there are eight constituent factors (F1 F8) of physical flexibility. Of those factors, the first concerns the flexibility of the trunk.

2) To choose the measurement items, it is valid to choose those items which showed a high factor loading to each factor. In this study, seven items (trunk flexion, upperarm raising, sideward neck bending, instep flexion and extension, wrist pronation and supination) were chosen.

3) From the results written above, it can be seen that it is inappropriate and insufficient to measure only the flexibility of the trunk. But to simplify the measurement method, physical flexibility can be estimated by measuring trunk flexion and sidewards bending.
\end{abstract}

(J. Physical Fitness Japan 1981, $30: 53 \sim 61$ )

\section{Introduction}

Larson L.A. ${ }^{16)}$ studied the structure of motor ability from three points, which were 1) fundamental structure and organic functions 2) fundamental motor elements 3) fundamental motor skills. Then he chose physical flexibility as one of the fundamental motor elements. Also Cureton T.K. ${ }^{5}$ found out by analyzing factors that besides the five factors, which were muscle strength, physical balance, agility, power and endurance there was a sixth factor of the factor structure of physical fitness. The sixth was physical flexibility. Furthermore Highmore G. ${ }^{14)}$ reports in his study by using factor analysis, that he found three group factors and a general factor of motor ability. Sills F. D. ${ }^{28}$ also reports, by using multivariate solution method, that physical flexibility is a factor of motor ability.

In 1945 O'Connor M.E. ${ }^{22)}$ like Cureton sug- gested six factors as the factor of physical fitness. Matsuda ${ }^{17)}$ also selected physical flexibility as well as balance and coordination of the body as factors of motor ability. Fleishman E.A. ${ }^{9)}$ considered that speed and flexibility have a motor ability factor in common. He classified this as extent flexibility and dynamic flexibility. But there still remains some difficulty in measuring dynamic flexibility. On the other hand, Matsuura ${ }^{18}$ ) used a hierarchical factor method to point out that as a secondary factor flexibilityendurance can be considered to be a motor ability item.

Both in Japan and in foreign countries, many studies were done on physical flexibility as an important constituent element of phyical fitness and motor ability.

Since the way to gain motor skill is to perform a movement efficiently, there is a need for smooth coordination of all parts of

\footnotetext{
* Institute of Health and Physical Education, College of General Education, Osaka University 1-1 Machikaneyama, Toyonaka, Osaka 560, Japan
} 
the body. Therefore each joint must efficiently contribute to the performance of the movement. To perform the movement efficiently, physical flexibility is necessary. This has a close relationship with the flexibility of the body joints. In the past, physical flexibility was considered only as a factor or a component of fundamental motor ability ${ }^{6}$, but there has been no study inquiring into the essence of physical flexibility alone. In a previous study, the author used the electrographical and roentgenological method ${ }^{23) 24}$ to investigate physical flexibility. Then this study was carried out to solve problems on physical flexibility using the statistical method. Customarily, in the past, to measure physical flexibility, the distance-method ${ }^{2021)}$ was used to measure trunk flexion and sit up and the degree-method was used to measure trunk extension and trunk sideward bend (left and right) ${ }^{25)}$. These were believed to be the items of physical flexibility but there are no studies investigating whether these are the representative items of measuring physical flexibility. This study investigates the validity of this method ${ }^{\text {7) }}$ and also solves some problems using the method written as follows. Therefore in this study as many measurable items as possible were considered and 19 items concerning joints of the body including trunk flexibility were measured. Then based on the measurements obtained, the factor analysis method ${ }^{1) ~ 4) 10) ~ 13) 15) 19) 29) ~ w a s ~ a p p l i e d, ~ w h i c h ~}$ will make examination of the factor structure of physical flexibility possible. Also in this study the validity of the method of measuring physical flexibility was investigated.

\section{Method}

1) Subject; 89 male students in the general education course of $\mathrm{K}$ University, Age 18 to 20 (average 19.2). These subjects were not chosen at random, so as not to bring about a variation in the factors chosen from the difference in subjects. This is characteristic of factor analysis.

2) Period ; From May to June 1967, after school.

3) Method ; Adopted the degree method ${ }^{25)}$ using a flexometer. All investigations on the objectivity and reliability of the degree method are reported in the previous study. ${ }^{26) 27)}$
In this study, for accurate measurements, all the measurements were personally performed and examined several times. And then using these data, the product moment correlation coefficient was calculated to seek the relationship between the first measurements and the second ones.

4) The measuring items were, four items to measure the neck-joint, (neck flexion, extension, neck sideward bend left and right), four for the wrist-joint (right and left, pronation and supination), two for the shoulderjoint (upperarm raising from the side, right and left), four for the instep-joint (flexion and extension of left and right ankle), and hip-joint, and then four for the flexibility of the trunk (trunk flexion, trunk extension, trunk sideward bend left and right). There were 19 items in total.

\section{Results and Discussion}

I. The examination on the reliability of the method

The results of the examination on the reliability using the test and re-test method in this study is shown in Table 1. Though the reliability coefficient of the 19 items has minimum of 0.80 in the item of right upperarm raising from the side, the others have a high coefficient of 0.82 and over. This can be considered fully appopriate, taking into consideration that the reliability of group measurement is 0.60 and over, and of individual measurement 0.80 and over.

II. The mean and the standard deviation of each item

Table 2 shows the mean and standard deviation of each item. From this, it can be seen that trunk flexion is about twice as much as trunk extension, and neck flexion is about half that of neck extension. There was a significant difference between trunk flexion and extension, between pronation and supination, and between instep flexion and extension. But there was not a significant difference between the right and left in the items of sideward bending of the neck, in pronation and supination, in instep flexion and extension, and in upperarm raising the difference being 0.05 . This means that in measuring the flexibility of the upper limbs and the lower limbs, it is necessary only to measure one of either 
Table 1. Reliability coefficient of nineteen test items

\begin{tabular}{l|c|l|c}
\multicolumn{1}{c|}{ test items } & $\mathrm{r}$ & \multicolumn{1}{|c|}{ test items } & $\mathrm{r}$ \\
\hline neck flexion & 0.95 & instep flexion (right) & 0.93 \\
neck extension & 0.87 & instep extension (right) & 0.83 \\
neck sideward bend (right) & 0.90 & instep flexion (left) & 0.91 \\
neck sideward bend (left) & 0.91 & instep extension (left) & 0.82 \\
wrist pronation (right) & 0.94 & hip joint & 0.85 \\
wrist supination (right) & 092 & trunk flexion & 0.96 \\
wrist pronation (left) & 0.94 & trunk extension & 0.86 \\
wrist supination (left) & 0.91 & trunk sideward bend (left) & 0.91 \\
upperarm raising from the side (right) & 0.80 & trunk sideward bend (right) & 0.88 \\
upperarm raising from the side (left) & 0.84 & & \\
\hline
\end{tabular}

Table 2. Means and standard deviations of measured nineteen test items

\begin{tabular}{l|r|r|l|r|r}
\multicolumn{1}{c}{ test items } & mean & SD & test items & mean & SD \\
\hline neck flexion & 44.0 & 5.9 & instep flexion (right) & 92.8 & 6.8 \\
neck extension & 84.2 & 4.6 & instep extension (right) & 162.2 & 5.2 \\
neck sideward bend (right) & 38.2 & 5.1 & instep flexion (left) & 94.5 & 72 \\
neck sideward bend (left) & 36.0 & 5.9 & instep extension (left) & 162.6 & 6.0 \\
wrist pronation (right) & 81.3 & 4.6 & hip joint & 123.8 & 8.9 \\
wrist supination (right) & 67.4 & 7.1 & trunk flexion & 130.2 & 11.1 \\
wrist pronation (left) & 81.9 & 4.9 & trunk extension & 65.4 & 8.8 \\
wrist supination (left) & 67.0 & 7.1 & trunk sideward bend (left) & 44.2 & 5.0 \\
upperarm raising (right) & 154.6 & 8.7 & trunk sideward bend (right) & 42.7 & 5.3 \\
upperarm raising (left) & 153.4 & 8.5 & & & \\
\hline
\end{tabular}

the right or left joint.

III. Factor analysis

All necessary calculations for analyzing factors in this study was done in the Kyoto University Data Processing Center using a HITAC 5020.

First of all, the relationship between the 19 items were calculated from the product moment correlation coefficient ${ }^{8)}$. Next, a correlation matrix was constructed having a diagonal element of 1.0000 . Table 3 shows the correlation matrix. Then by applying the incomplete principal component solution method to the correlation matrix, a pattern matrix was constracted by computing the eigen value and the factor loading of each factor. By this matrix, those which were larger than 1.0000 , and the eigen values having approximately this figure were picked up from the 19 eigen values and were also considered to explain $70 \%$ of the total variance. In this occassion, the eigen value more than 0.9100 were used. So there are eight factors which explains the $72 \%$ of the total variance.

Table 4 shows the eigen value of the eight elements chosen and the factor loading of each item. But because these figures show the unroted factor loading in the process of analyzing factors, it is difficult to interpret the eight factors chosen. So, to make the interpretation less difficult or in other words, to make the structure of the factor pattern matrix simpler, orthogonal rotation was done to these factor axis by the normal varimax criterion method. Table 5 shows the figures obtained by the rotation, which is the factor loading after rotation. The factor pattern matrix after the rotation is believed to have 
Table 3. Correlation matrix

\begin{tabular}{r|l|rrrrrr}
\hline No. & \multicolumn{1}{|c|}{ test items } & \multicolumn{1}{c}{1} & \multicolumn{1}{c}{2} & \multicolumn{1}{c}{3} & \multicolumn{1}{c}{4} & \multicolumn{1}{c}{6} \\
\hline 1 & neck flexion & 1.0000 & & & & & \\
2 & neck extension & 0.2258 & 1.0000 & & & & \\
3 & neck sideward bend (right) & 0.1761 & 0.0742 & 1.0000 & & & \\
4 & neck sideward bend (left) & 0.1516 & 0.2550 & 0.8174 & 1.0000 & & \\
5 & wrist pronation (right) & 0.2350 & -0.0098 & 0.1122 & 0.0193 & 1.0000 & \\
6 & wrist supination (right) & 0.1722 & 0.1377 & 0.1701 & 0.2206 & 0.3026 & 1.0000 \\
7 & wrist pronation (left) & 0.2639 & 0.0724 & 0.1017 & 0.2030 & 0.3837 & 0.1143 \\
8 & wrist supination (left) & 0.3093 & 0.2091 & 0.2958 & 0.3428 & 0.2219 & 0.5924 \\
9 & upperarm raising (right) & 0.0193 & 0.2498 & 0.0931 & 0.1961 & 0.1003 & 0.3174 \\
10 & upperarm raising (left) & 0.0232 & 0.1303 & 0.0697 & 0.1176 & 0.1078 & 0.2012 \\
11 & instep flexion (right) & -0.0149 & -0.2226 & 0.0183 & -0.0782 & -0.0461 & -0.1590 \\
12 & instep extension (right) & -0.0042 & -0.0670 & 0.0802 & 0.0393 & 0.0416 & 0.1486 \\
13 & instep flexion (left) & -0.0655 & -0.2586 & -0.0508 & -0.1163 & 0.0872 & 0.2532 \\
14 & instep extension (left) & 0.0857 & -0.1519 & 0.0773 & -0.0664 & 0.1665 & 0.1031 \\
15 & hip joint & 0.1173 & 0.2599 & 0.0034 & 0.1092 & 0.1880 & 0.0607 \\
16 & trunk flexion & 0.1639 & 0.1599 & 0.0975 & 0.0764 & 0.1419 & 0.0654 \\
17 & trunk extension & 0.0968 & 0.0412 & -0.0407 & -0.0021 & 0.1785 & 0.0622 \\
18 & trunk sideward bend (left) & 0.1786 & 0.1278 & 0.1678 & 0.1815 & -0.0159 & 0.0935 \\
19 & trunk sideward bend (right) & 0.1420 & 0.1390 & 0.1577 & 0.2127 & 0.0315 & 0.1519 \\
\hline
\end{tabular}

Table 4. Unrotated factor loadings of the eight factors

\begin{tabular}{l|r|r|r|r|r|r|r|r}
\hline test items & F1 & F2 & F 3 & F & F 5 & F6 & F 7 & F 8 \\
\hline 1 neck flexion & 0.3274 & 0.0000 & 0.0000 & -0.0323 & 0.0000 & 0.0000 & -0.0215 & 0.9441 \\
2 neck extension & 0.9872 & 0.0000 & 0.0000 & -0.0973 & 0.0739 & 0.0000 & -0.0649 & -0.0782 \\
3 neck sideward bend (right) & 0.3713 & 0.5507 & 0.7451 & -0.0366 & 0.0278 & 0.0000 & -0.0244 & -0.0294 \\
4 neck sideward bend (left) & 0.3713 & 0.5507 & 0.7451 & -0.0366 & 0.0278 & 0.0000 & -0.0244 & -0.0294 \\
5 wrist pronation (right) & 0.2824 & 0.0000 & 0.0000 & 0.0000 & 0.0000 & 0.0000 & 0.9593 & 0.0000 \\
6 wrist supination (right) & 0.3637 & 0.5600 & -0.3510 & -0.0358 & 0.0272 & 0.6538 & -0.0239 & -0.0288 \\
7 wrist pronation (left) & 0.9198 & -0.3632 & -0.0046 & -0.0906 & 0.0689 & 0.0000 & -0.0605 & -0.0729 \\
8 wrist supination (left) & 0.3637 & 0.5600 & -0.3510 & -0.0358 & 0.0272 & 0.6538 & -0.0239 & -0.0288 \\
9 upperarm raising (right) & 0.4120 & 0.6345 & -0.3976 & -0.0406 & 0.0309 & -0.5149 & -0.0271 & -0.0326 \\
10 upperarm raising (left) & 0.4120 & 0.6345 & -0.3976 & -0.0406 & 0.0309 & -0.5149 & -0.0271 & -0.0326 \\
11 instep flexion (right) & -0.5183 & 0.2047 & 0.0026 & 0.0511 & 0.8270 & 0.0000 & 0.0341 & 0.0411 \\
12 instep extension (right) & 0.2667 & 0.0000 & 0.0000 & 0.9636 & 0.0000 & 0.0000 & -0.0175 & 0.0000 \\
13 instep flexion (left) & -0.3724 & 0.0000 & 0.0000 & 0.0367 & 0.9265 & 0.0000 & 0.0245 & 0.0295 \\
14 instep extension (left) & 0.2667 & 0.0000 & 0.0000 & 0.9636 & 0.0000 & 0.0000 & -0.0175 & 0.0000 \\
15 hip joint & 0.9058 & -0.3577 & -0.0045 & -0.0892 & 0.1866 & 0.0000 & -0.0596 & -0.0718 \\
16 trunk flexion & 0.9058 & -0.3577 & -0.0045 & -0.0892 & 0.1866 & 0.0000 & -0.0596 & -0.0718 \\
17 trunk extension & 0.9057 & -0.3577 & -0.0045 & -0.0892 & 0.1866 & 0.0000 & -0.0597 & -0.0718 \\
18 trunk sideward bend (left) & 0.9058 & -0.3576 & -0.0046 & -0.0893 & 0.1867 & 0.0000 & -0.0596 & -0.0718 \\
19 trunk sideward bend (right) & 0.9058 & -0.3576 & -0.0046 & -0.0893 & 0.1867 & 0.0000 & -0.0596 & -0.0718 \\
\hline eigen values & 4.1970 & 2.2020 & 1.4783 & 1.4660 & 1.3285 & 1.1348 & 0.9380 & 0.9158 \\
\hline
\end{tabular}


(inter-correlations)

\begin{tabular}{|c|c|c|c|c|c|c|c|c|c|c|c|c|}
\hline 7 & 8 & 9 & 10 & 11 & 12 & 13 & 14 & 15 & 16 & 17 & 18 & 19 \\
\hline
\end{tabular}

\begin{tabular}{|c|c|c|c|c|c|c|c|c|}
\hline 1. 0000 & & & & & & & & \\
\hline 0.3418 & 1.0000 & & & & & & & \\
\hline 0.1272 & 0.2624 & 1.0000 & & & & & & \\
\hline 0.1152 & 0.3167 & 0.7931 & 1.0000 & & & & & \\
\hline-0.1966 & -0.0661 & -0.1993 & -0.0778 & 1.0000 & & & & \\
\hline-0.1880 & -0.0843 & -0.0179 & 0.0762 & 0.0477 & 1.0000 & & & \\
\hline 0.0349 & -0.1152 & -0.1636 & -0.0791 & 0.5674 & -0.0990 & 1.0000 & & \\
\hline 0.0863 & 0.0105 & 0.0673 & 0.2016 & 0.1308 & 0.5369 & 0.1307 & 1.0000 & \\
\hline 0.3348 & 0.0681 & 0.2508 & 0.2003 & -0.2188 & 0.1500 & -0.1162 & 0.18051 .0000 & \\
\hline 0.1785 & 0.1155 & 0.0537 & 0.0438 & -0.2782 & 0.1968 & -0.1602 & $0.12920 .5341 \quad 1.0000$ & \\
\hline 0.3615 & 0.0549 & 0.1361 & 0.0547 & -0.2888 & 0.1712 & -0.0816 & $\begin{array}{llll}0.2364 & 0.5229 & 0.5626 & 1.0000\end{array}$ & \\
\hline 0.1437 & 0.0294 & 0.1614 & 0.1021 & -0.2615 & 0.1305 & -0.0744 & $\begin{array}{lllll}0.1886 & 0.3141 & 0.4919 & 0.4660\end{array}$ & 1.0000 \\
\hline 0.2650 & 0.1044 & 0.1600 & 0.1012 & -0.3617 & 0.0708 & -0.1447 & $\begin{array}{llll}0.1385 & 0.3572 & 0.5349 & 0.5467\end{array}$ & 70.86831 .0000 \\
\hline
\end{tabular}

Table 5. Rotated factor pattern matrix

\begin{tabular}{|c|c|c|c|c|c|c|c|c|}
\hline test items factors & $\mathrm{F} 1$ & $\mathrm{~F} 2$ & F 3 & $\mathrm{~F} 4$ & F 5 & $\mathrm{~F} 6$ & F 7 & F 8 \\
\hline 1 neck flexion & .17512 & .04779 & .04414 & .02214 & -.04651 & .04134 & .01975 & .97997 \\
\hline 2 neck extension & .31252 & .09200 & .08433 & .02086 & -.06205 & .07823 & .01741 & .02018 \\
\hline 3 neck sideward bend (right) & 09568 & .08841 & .93933 & .02349 & -.02879 & .07670 & .02021 & 02361 \\
\hline 4 neck sideward bend (left) & .09428 & .08644 & .93930 & .02333 & -.02780 & .07667 & .02017 & .02359 \\
\hline 5 wrist pronation (right) & .14799 & .04037 & .03734 & .02553 & -.03927 & .03496 & .98554 & .01941 \\
\hline 6 wrist supination (right) & .08380 & .13117 & .07258 & .02094 & -.02555 & .86701 & .01821 & .02120 \\
\hline 7 wrist pronation (left) & .43199 & .01611 & .01508 & .02375 & -08282 & .01442 & .01957 & .02265 \\
\hline 8 wrist supinaion $(\mathrm{left})$ & .03380 & .13066 & .07222 & .02087 & -.02555 & .86699 & .01819 & .02117 \\
\hline 9 upperarm raising (right) & .10206 & .91481 & .08899 & .02554 & -.03117 & .13998 & .02240 & .02612 \\
\hline 10 upperarm raising (left) & .10215 & .91469 & .08861 & .02538 & -.03120 & .13987 & .02236 & .02605 \\
\hline 11 instep flexion (right) & -.28544 & .00468 & .00385 & -.02755 & .81237 & .00294 & -.02342 & -.02717 \\
\hline 12 instep extension (right) & .08704 & .02336 & .02132 & .87092 & -.02284 & .02028 & .01279 & .01106 \\
\hline 13 instep flexion (left) & -.08394 & -.06330 & -.05814 & -.02193 & .89652 & -.05411 & -.01911 & -.02223 \\
\hline 14 instep extension (left) & .08704 & .02385 & .02132 & .87101 & -.02284 & .02030 & .01283 & .01106 \\
\hline 15 hip joint & 65358 & .02598 & .02426 & .03469 & .89652 & .02312 & .02851 & .03298 \\
\hline 16 trunk flexion & .71017 & .02823 & .02636 & .03770 & -.04985 & .02513 & .03098 & .03585 \\
\hline 17 trunk extension & .74289 & .02953 & .02758 & .03943 & -.05214 & .02628 & .03240 & .03750 \\
\hline 18 trunk sideward bend (left) & .88539 & .03520 & .03287 & .04699 & -.06162 & .03133 & .03862 & .04473 \\
\hline 19 trunk sideward bend (right) & .88466 & .03546 & .03279 & .04627 & -.06214 & .03128 & .03856 & .04469 \\
\hline eigen values & 2.9160 & 2.3083 & 1.7472 & 1.5760 & 1. 4084 & 1. 3125 & 1.1970 & 1.1843 \\
\hline degree of contribution $\left(0_{0}^{\prime}\right)$ & 15.35 & 12.15 & 9.20 & 8.29 & 7.41 & 6.91 & 6.30 & 6.23 \\
\hline
\end{tabular}


become quite simple. Therefore, in this factor pattern matrix, interpretation of each factor and of each item measured was attempted.

IV. The evaluation of the factor

The first factor: The first factor is so defined by all items to have the largest contribution to the total variance. For the first factor having such a characteristic, there were five items (trunk-flexion, extension, right and left sideward bend, and hip-joint) which showed a significant factor loading. Other than these five, there were the extension of the neck-joint and the pronation of the wristjoint which showed quite an amount of factor loading. But these were not significant at the level of 0.05 . Therefore, these factors were considered to have a common factor with the five items mentioned above. And these five occupy $62.9 \%$ (mean factor loading after $z$ transformation; $0.793^{2} \times 100$ ) of the total variance of the first factor. This means that the five items, trunk-flexion, extension, right and left sideward bend and hip-joint, explains $62.9 \%$ of the contribution amount defined by the first factor. These items (except for the hip-joint) are the same with the measuring items which have been customarily using the degree method. From this analysis, the addition of the hip-joint item was recognized to be valid. So these main factors can be interpreted as the factor of trunk flexibility.

The second factor: This factor shows a very high factor loading only in both the upperarm raising from the right and the upperarm raising from the left. Other than these two, only a very low factor loading can be seen. Therefore, the second factor has a close relationship with these two items. The two items show a contribution of $83.7 \%$ of the total variance. So the item is interpreted to be a factor measuring the shoulder-joint flexibility.

The third factor: This factor shows the highest factor loading of 0.9393 in the item of the sideward bending of the neck-joint, for both left and right. The amount of contribution of the two items to the third factor is $88.2 \%$. Therefore, it can be said that these two items explain about $88 \%^{\circ}$ of the contribution amount defined by this factor. But also, only a small factor loading of 0.0441 and 0.0843 is shown for the flexion and extension of the neck-joint. Further study must be done in the future on this subject but these two were considered to have a separate factor from the others. Anyway, it is probably appropriate to define this factor as the neckjoint capacity.

The fourth factor: This factor shows a significant factor loading only to the two items of the extension of the left and right instep. The factor loading of these two items are far higher than any other items. Also the amount of contribution of the fourth factor to the total variance is $75.9 \%$. Similar to the third factor, here too, the ability of extension and flexion of the instep-joint can be recognized as a independent factor. Therefore, it can be defined as the factor concerning the extension and flexion of the instep-joint.

The fifth factor: Contrary to the fourth factor, this factor shows a high factor loading with an average of 0.8722 concerning the flexion of the two items, the left and right instep-joint. It is characteristic that all other factors show a negative factor loading. The amount of contribution to the total variance by these two factors is $76.0 \%$. This is almost equivalent to the cases of extension. Therefore, it can be defined as the flexion factor of the instep-joint.

The sixth factor: The two items, the supination of the right and left wrist-joint showed a high positive factor loading. Similar to the seventh factor mentioned below, the pronation and supination of the wrist-joint does not seem to have a factor in common. The amount of contribution of the supination of the wrist-joint to the total variance was $75.2 \%$. From this we can easily define this factor as the factor concerning the supination of the wrist-joint.

The seventh factor: This factor showed a very high factor loading of 0.9855 only for the pronation of the right wrist-joint. It is diffcult to comprehend the fact that it does not share a common factor with the left wristjoint. Anyway, the pronation of the right wrist-joint explains $97.2 \%$ of the total variance defined by the seventh factor. This factor can be defined as the pronation factor of the wrist-joint.

The eighth factor: This factor showed a very high positive factor loading only to neck 
Table 6. Isolated the eight factors

\begin{tabular}{l|l}
\hline F 1 & factor concerning the flexibility of the trunk \\
\hline F 2 & factor concerning the flexibility of the shoulder-joint \\
\hline F 3 & factor concerning the sideward bend of the neck-joint \\
\hline F 4 & factor concerning the extension of the instep-joint \\
\hline F 5 & factor concerning the flexion of the instep-joint \\
\hline F 6 & factor concerning the supination of the wrist-joint \\
\hline F 7 & factor concerning the pronation of the wrist-joint \\
\hline F 8 & factor concerning the flexion of the neck-joint
\end{tabular}

Table 7. Selected measuring items for physical flexibility

\begin{tabular}{c|c||c|c}
\hline factors & selected measuring items & factors & selected measuring items \\
\hline F 1 & trunk flexion & F 5 & left instep flexion \\
\hline F 2 & right upperarm raising & F 6 & right wrist supination \\
\hline F 3 & right neck sideward bend & F 7 & right wrist pronation \\
\hline F 4 & left instep extension & F 8 & neck flexion \\
\hline
\end{tabular}

flexion. The factor loading of neck extension is low. It may be appropriate to define this factor as the factor concerning the neck flexion capacity, because the amount of contribution to this factor by neck flexion is 96.0 $\because$.

As mentioned above the eight factors chosen in this study can be written as in Table 6 .

$\mathrm{V}$. Items to measure physical flexibility

As mentioned in the discussion, the constituent factors of flexibility has been found. Therefore, to determine which items measure flexibility, from the constituent factors already defined, only those items that can be measured must be chosen. This means that it is appropriate to choose each factor and those items which have the highest factor loading. Table 7 shows the items chosen using this method. The validity is about $72 \%$.

Concerning the first factor, as shown in the result and discussion, it is appropriate to use the items, the sideward bending of both right and left, which showed the highest factor loading. The validity lessens when we take into consideration the utility and the reliability of the measuring method, but trunk flexion can still be chosen for the first factor.
The reliability of all the measuring items of the second through the eighth factor shows more than $0.80(r)$. To measure physical flexibility, it is insufficient only to measure the flexibility of the trunk. It has been suggested that it is valid to include the seven items mentioned above. Taking into consideration simplicity and also when it is necessary to measure a large sample, it can be recognized that it is possible to estimate flexibility by measuring the flexion and extension of the trunk.

\section{Summary}

In this study, 19 joints which have close relationship with flexibility were chosen from each part of the body. The constituent factors of flexibility was examined by applying the factor analysis method. Then, this study on the validity of using the measuring items of physical flexibility from their factor construction was made. And also the validity of using trunk flexion, extension, sideward bending left and right, and sit-up as the typical items of measuring flexibility were experimented and studied.

1) There were eight factors identified 
concerning physical flexibility. They were, (1) the factor concerning the flexibility of the trunk (2) the factor concerning the flexibility of the shoulder-joint (3) the factor concerning the sideward bend of the neck-joint (4) the factor concerning the extension of the instep-joint (5) the factor concerning the flexion of the instep-joint (6) the factor concerning the supination of the wrist-joint (7) the factor concerning the pronation of the wrist-joint (8) the factor concerning the flexion of the neck-joint.

2) Therefore, to measure flexibility, it is valid to choose those factors and those items which showed the highest factor loading to the factor chosen. As a result, there were seven items chosen, which were, trunk flexion, right upperarm raising from the side, sideward bending of the neck-joint, flexion and extension of the instep-joint, and pronation and supination of the right wrist-joint. It can now be seen that it is inappropriate to measure only the flexibility of the trunk as we have been doing.

3) However when measuring a large sample and when there is a need for a simple method, physical flexibility can be measured by measuring two items, trunk flexion and trunk extension. In this case the validity may lessen but by considering the amount of contribution of the main factor, it is possible to estimate flexibility.

Finally, this study was funded by the Japanese Ministry of Education in 1967.

(Received for publication August 6, 1980)

\section{References}

1) Carroll, John B. and Schweiker, Robert F., (1951) : Factor analysis in educational research, Rev. Ed. Res., 21, 368-388.

2) Comrey, Andrew L., (1967): A journal devoted to the development of psychology as a quantitative rational science, Psychometrika, 32, 143-154.

3) Coombs, Clyde H., (1964): A theory of data, John Wiley \& Sons, Inc., New York, 181-192.

4) Cronbach, Lee J., (1960) : Essentials of psychological testing, Happer \& Brothers, Publishers, New York, 214-268.

5) Cureton, Thomas K., (1947) : Physical fitness appraisal and guidance, The C.V.
Mosby Co., St. Louis, pp. 558.

6) Dimock, H.S., (1935) : A research in adolescence and physical growth, Child Development, 6, 177-195.

7) Ebel, Robert L., (1965) : Measuring educational achivement, Prentice-Hall, Inc., New Jersey, 376-395.

8) Ferguson, George A., (1959) : Statistical analysis in psychology and education, Mc Graw-Hill, New York, 86-98.

9) Fleishman, E.A. and Nicks, D.C., (1962): What do physical fitness tests measure?, Educational and Psychological Measurement, 22(1), 77-96.

10) Garrett, Henry E. and Woodworth, R.S., (1958) : Statistics in psychology and education, Longmans, Green Co., New York, 277 -308 .

11) Guttman, Louis, (1952) : Multiple group methods for common-factor analysis. Psychometrika, 17, 209-222.

12) Guttman, Louis, (1944): General theory and methods for matric Factoring, Psychometrika, 9, 1-16.

13) Harman, Harry H., (1960) : Modern factor analysis, The University of Chicago Press, U.S.A., 154-191, 289-308.

14) Highmore, G., (1956) : A factor analysis of athletic ability, Research Quarterly, 27 (1), 1-11.

15) Holzinger, K.T., (1944): A simple method of factor analysis, Psychometrika, 9, 257262.

16) Larson, Leonard A., (1941) : A factor analysis of motor ability variables and tests, with tests for college men, Research Quarterly, 12, 499-517.

17) Matsuda, Iwao, (1961): Development of motor ability in infancy, Tokyo University of Education Press, Tokyo, 1, 38-58.

18) Matsuura, Yoshiyuki, (1968) : Structure of factors in motor ability, Fumaido Press, Tokyo, 362-363.

19) McNemar, Quinn, (1962) : Psychological statistics, John Wiley and Sons, Inc., New York, 252-287.

20) Ministry of Education, (1964) : Methods of sports-test, Japanese Ministry of Education Tokyo.

21) Nunnally, Jum C.,(1959) : Tests and measurements, McGraw-Hill Book Co., New York, 141-162.

22) O'Connor, M.E., and Cureton, T.K., (1945): Motor fitness tests for high school girls, Research Quarterly, 16, 302-314.

23) Ohyama, Yoshinori, (1968) : Electromyogra- 
phical study on the flexibility of the human body, J. School Health and Medicine, 10 (3), 1-9.

24) Ohyama, Yoshinori, (1967) : Roentgenological study on the flexibility of the human body, J. Physical Fitness Japan, 15(3), 120 -127 .

25) Ohyama, Yoshinori, (1965): Hygienic study on the flexibility of the normal : part 1 , Jap. J. Hyg., 20(2), 25-30.

26) Ohyama, Yoshinori, (1966): Hygienic study on the flexibility of the normal : part 2 ,
Jap. J. Hyg., 21 (2), 38-45.

27) Ohyama, Yoshinori, (1966): Hygienic study on the flexibility of the normal : part 3 , Jap. J. Hyg., 21(5), 20-25.

28) Sills, F.D., (1950) : A factor analysis of somatotypes and their relationship to achievement in motor skills, Research Quarterly, 21(4), 424-437.

29) Tucker, L.R.,(1940) : The role of correlated factors in factor analysis, Psychometrika, 5, 141-152. 